Objectives To provide opportunities for sharing experiences and discussing lessons learned in the use of CDSSs in different countries.

Target Group Guideline developers, guideline implementers, clinicians, researchers, policy makers.

Moderator Jako Burgers, Dutch College of General Practitioners (NHG), The Netherlands Invited speakers: Marjolein Lugtenberg, IQ healthcare, Radboud University Nijmegen Medical Centre, The Netherlands. Linn Brandt, Inlandet Hospital Trust/ University of Oslo, Norway. Ilkka Kunnamo, Duodecim Medical Publications Ltd./University of Helsinki, Finland. Richard Shiffman, Center for Medical Informatics, Yale University, USA.

Description of Session and Speaker Topics In this session initiatives on CDSSs from four different countries (The Netherlands, Norway, Finland, and the USA) will be presented, each taking a different perspective on the use of CDSSs to improve the uptake of guidelines. Issues that will be considered are how to deal with various alerts within multiple disease areas, distinguishing alerts from strong and weak guideline recommendations, and creating composite views of data and recommendations. Finally, conclusions are drawn on the strengths and weaknesses of developing, implementing, and evaluating each system and lessons learned will be discussed with the audience.

\section{PS CHALLENGES AND OPPORTUNITIES IN LOW-RESOURCE SETTINGS: GUIDELINE DEVELOPMENT, ADAPTATION IMPLEMENTATION AND PERFORMANCE MEASUREMENT}

${ }^{5,4}$ S Huckson, ${ }^{1} \mathrm{M}$ Beena, ${ }^{2} \mathrm{~N}$ Opiyo, ${ }^{3,4}$ E Lang, ${ }^{6,7} \mathrm{M}$ Koster. ${ }^{1}$ National Rural Health Mission, Kerala, India; ${ }^{2}$ KEMRI-Wellcome Trust Research Programme, Nairobi, Kenya; ${ }^{3}$ Alberta Health Services, University of Calgary, Calgary, Alberta, Canada; ${ }^{4}$ G-I-N Emergency Care Community, National Health and Medical Research Council's National Institute of Clinical St. Melbourne Australia; ${ }^{5}$ Kaiser Permanente Southern California Pasadena, CA US G-I-N North America Steering Group, US

\section{0:1136/bmjqs-2013-002293.18}

Background Health care and health policy organisations seeking to improve medical care in low-resource settings with significant numbers of uninsured, medically underserved and/or low-income populations face significant challenges in developing, adapting, implementing and monitoring improvement with clinical practice guidelines.

Objectives/Goals To provide opportunities for panel members and conference participants to exchange experiences, challenges, lessons learned, and opportunities for collaboration related to guideline development, adaptation, implementation and performance measurement in low-resource settings.

Target Group, Suggested Audience Those involved in guideline development, adaptation, implementation and/or performance measurement activities, especially in medically underserved, lowincome and low-resource settings.

Description of Session and Speaker Topics The session will include brief speaker presentations, followed by interactive, facilitated discussion between panellists and audience members. Dr. Beena will discuss development of guideline-based quality standards to decrease maternal mortality in rural settings in Kerala, India; Dr. Opiyo will discuss guideline adaptation and implementation efforts in Nairobi to improve child and newborn health; Dr. Lang will discuss the G-I-N Emergency Care Community's efforts to support collaboration across the field of international emergency care, including adaptation of sepsis guidelines for global use in low-resource settings. Sue Huckson will moderate the discussion.

\section{PS INCORPORATING GUIDELINES INTO LOCAL CLINICAL PRACTICE AND POLICY THROUGH THE USE OF PRACTICE-BASED HEALTH TECHNOLOGY ASSESSMENT}

${ }^{1,2,3} \mathrm{C}$ Umscheid, ${ }^{4} \mathrm{M}$ Koster, ${ }^{5,6} \mathrm{M}$ Marchetti, ${ }^{7,8,9} \mathrm{M}$ Helfand, ${ }^{3,10} \mathrm{P}$ Wyer. ${ }^{1}$ Penn Medicine Center for Evidence-based Practice, Philadelphia, PA, USA ECRI - Penn; ${ }^{2}$ AHRQ Evidencebased Practice Center, Philadelphia, PA, USA; ${ }^{3}$ Section of Evidence Based Health Care (SEBHC), New York Academy of Medicine, NY, USA; ${ }^{4}$ Technology Assessment and Guidelines Unit, Kaiser Permanente, Pasadena, CA, USA; ${ }^{5}$ HTA Unit of the Catholic University Hospital, Rome, Italy; ${ }^{6}$ HTA International Interest Group on Hospital Based HTA Edmonton, Alberta, Canada; ${ }^{7}$ Veterans Affairs Evidence-based Synthesis Program Portland, OR, USA; ${ }^{8}$ Scientific Resource Center, AHRQ EPC Program Portland, OR, USA; ${ }^{9}$ Oregon Health and Sciences University Portland, OR, USA; ${ }^{10}$ Columbia University College of Physicians and Surgeons NY, USA

\section{0:1136/bmjqs-2013-002293.19}

Background Practice-based health technology assessment (PBHTA) has the potential to improve the quality, safety and value of patient care by synthesising evidence to provide timely guidance for clinical practice, policy, formulary management, operations, and purchasing decisions. Hospital-based HTA centres are active in Western Europe and Canada, but less so in the US, and some operate in conjunction with formal evidence-based guideline programmes.

Objectives/Goals To actively engage leaders of PB-HTA to discuss strengths and limitations, lessons learned, and the role of PB-HTA in the development, dissemination, and implementation of guidance within health care systems.

Target Group/Suggested Audience Senior executives/administrators, and clinical policy, quality and safety leaders in healthcare organisations and networks who develop, implement and measure performance related to clinical guidance.

Description of Aession and Speaker Topics Dr. Wyer, who leads a PB-HTA capacity building programme for health care organisations at the NYAM SEBHC, will engage the panellists in a discussion of their experiences leading PB-HTA efforts at Kaiser Permanente (Ms. Koster), the Veterans Administration (Dr. Helfand), Penn Medicine (Dr. Umscheid) and the HTA unit at the Catholic University Hospital in Rome, Italy (Dr. Marchetti). The discussion will address the potential for guidance developed by PB-HTA centres to impact the quality, safety and value of patient care, similarities and differences in national and international efforts, and future directions for the field.

\section{PS SETTING NEW HORIZONS IN OPTIMIZING GUIDELINE UTILITY}

II Scott, ${ }^{2} \mathrm{~S}$ Norris, ${ }^{3} \mathrm{H}$ Schünemann, ${ }^{3} \mathrm{G}$ Guyatt. ${ }^{1}$ Internal Medicine and Clinical Epidemiology, Princess Alexandra Hospital, Brisbane, Australia; ${ }^{2}$ Guideline Review Committee, World Health Organisation, Geneva, Switzerland; ${ }^{3}$ Department of Clinical Epidemiology and Biostatistics, McMaster University, Hamilton, Canada

\section{0:1136/bmjqs-2013-002293.20}

Background To maximise uptake, CPG recommendations must avoid of bias and be responsive to the needs of clinicians and patients from different populations and settings.

Objectives/Goal To discuss three key challenges to CPG use: 1) building consensus and minimising conflicts of interest in formulating recommendations for specific patient populations; 2) taking account of patient multi-morbidity; 3) incorporating patient values and preferences for specific outcomes.

Target group Suggested audience Guideline developers and writing groups, clinical researchers, users of guidelines (clinicians, patients). 